\title{
Electron beam studies of light collection in a scintillating counter with embedded fibers
}

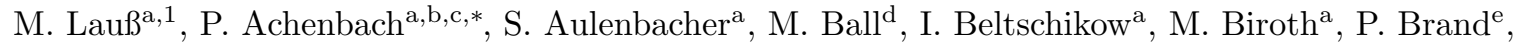

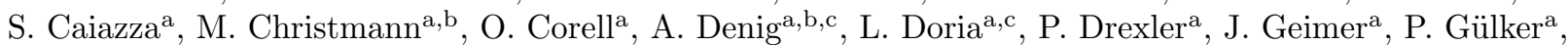 \\ T. Kolar ${ }^{\mathrm{f}}$,W. Lauth ${ }^{\mathrm{a}}$, M. Littich ${ }^{\mathrm{a}}$, M. Lupberger ${ }^{\text {h }}$, S. Lunkenheimer ${ }^{\mathrm{a}}$, D. Markus ${ }^{\mathrm{a}}$, M. Mauch $^{\mathrm{b}}$, \\ H. Merkel ${ }^{a, c}$, M. Mihovilovič́f,g, J. Müller ${ }^{\mathrm{a}}$, B. S. Schlimme ${ }^{\mathrm{a}}$, C. Sfientia ${ }^{\mathrm{a}, \mathrm{c}}$, S. Šrrca ${ }^{\mathrm{f}, \mathrm{g}}$, S. Stengel $^{\mathrm{a}}$, \\ C. Szyszka ${ }^{\mathrm{a}}$, S. Vestrick ${ }^{\mathrm{e}}$, \\ for the MAGIX Collaboration

\footnotetext{
${ }^{a}$ Institut für Kernphysik, Johannes Gutenberg-Universität, J.-J.-Becherweg 45, D-55099 Mainz, Germany

${ }^{b}$ Helmholtz Institute Mainz, GSI Helmholtzzentrum für Schwerionenforschung, Darmstadt, Johannes Gutenberg-Universität, D-55099 Mainz, Germany

${ }^{c}$ PRISMA ${ }^{+}$Cluster of Excellence, Johannes Gutenberg-Universität, Staudingerweg 9, D-55128 Mainz, Germany

${ }^{d}$ Helmholtz-Institut für Strahlen- und Kernphysik, Rheinische Friedrich-Wilhelms-Universität Bonn, Nussallee 14-16, D-53115 Bonn, Germany

${ }^{e}$ Institut für Kernphysik, Westfälische Wilhelms-Universität Münster, Wilhelm-Klemm-Str. 9, D-48149 Münster, Germany ${ }^{f}$ Jožef Stefan Institute, SI-1000 Ljubljana, Slovenia

${ }^{g}$ Faculty of Mathematics and Physics, University of Ljubljana, SI-1000 Ljubljana, Slovenia
} \\ ${ }^{h}$ Physikalisches Institut, Rheinische Friedrich-Wilhelms-Universität, Nussallee 12, D-53115 Bonn, Germany
}

\begin{abstract}
The light collection of several fiber configurations embedded in a box-shaped plastic scintillating counter was studied by scanning with minimum ionizing electrons. The light was read out by silicon photomultipliers at both ends. The light yield produced by the $855-\mathrm{MeV}$ beam of the Mainz Microtron showed a strong dependence on the transverse distance from the beam position to the fibers. The observations were modeled by attributing the collection of indirect light inside of the counter and of direct light reaching a fiber to the total light yield. The light collection with fibers was compared to that of a scintillating counter without fibers. These studies were carried out within the development of plastic scintillating detectors as an active veto system for the DarkMESA electron beam-dump experiment that will search for light dark matter particles in the $\mathrm{MeV}$ mass range.
\end{abstract}

Keywords: Plastic scintillating counter, Wavelength-shifting fiber, Light yield, Silicon photomultiplier (SiPM), Electron beam tests

${ }^{*}$ Corresponding author at: Institut für Kernphysik, J.J.-Becherweg 45, Johannes Gutenberg-Universität, D-55099 Mainz, Germany. Tel.: +49 613139 25777; Fax: +49 6131 3922964.

Email address: achenbach@uni-mainz.de (P. Achenbach)

${ }^{1}$ Part of master thesis.

Preprint submitted to Nucl. Instrum. Methods Phys. Res. A

\section{Introduction}

The Johannes Gutenberg University Mainz is currently constructing the new continuous-wave multiturn electron linac MESA (Mainz Energy Recovering Superconducting Accelerator) on the Gutenberg Campus [1]. For the DarkMESA experiment, the high-power beam dump of the accelerator will be used 
as a target for the possible production of dark sector particles in the $\mathrm{MeV}$ mass range $[2,3]$. Once discovered, these could provide information on the structure of dark matter, which makes up a large proportion of our universe [4].

The detector concept of the DarkMESA experiment will implement electromagnetic calorimeters surrounded by active veto counters. The calorimeters will detect the transferred energy in elastic scattering of the dark sector particles by atomic-shell electrons [5], in which the energy range is defined by the $150 \mathrm{MeV}$ energy of the electron beam. The detector site will be heavily shielded from the beam, blocking practically all beam-related Standard Model particles. It is crucial for this experiment that cosmogenic particles leading to background events are vetoed with a high detection efficiency and homogeneity.

In nuclear and particle physics, the combination of wavelength-shifting (WLS) fibers with a silicon photomultiplier (SiPM) readout is known to be a viable option for the operation of a scintillation counter since the first proofs of concept, e.g. in Refs. [6, 7], and the pioneering applications in large numbers by the CALICE [8] and T2K Collaborations [9, 10]. Today, it is an efficient and robust technique that has found a widespread use in the construction of tracking detectors and calorimeters, see for instance the recent review on this branch of instrumentation in Ref. [11]. A number of studies exist on the photoelectron yields for different geometries and setups of scintillation counters with embedded WLS fibers, see e.g. Refs. [12, 13].

The DarkMESA experiment requires a highly efficient, compact, and cost-effective veto system which is capable of muon identification and also accommodates suppression of gamma-ray and neutron background. The planned veto detector system will consist of approximately 80 box-shaped plastic scintillating counters, each of $2 \mathrm{~cm}$ thickness and having a maximum size of $25 \times 200 \mathrm{~cm}^{2}$. The counters will be arranged in two layers, and read out by SiPMs, the latter possibly connected to WLS fibers for an enhanced light collection. Sheets of lead, between the inner and the outer veto layer, should prevent low-energy $\gamma$-rays from reaching the calorimeter. In a Monte Carlo simulation using Geant4 and its internal optical transport routine, such scintillation counters without WLS fibers showed a light yield of 15 photoelectrons (p.e.) per $\mathrm{MeV}$ energy deposition, also for the longest detector element of $200 \mathrm{~cm}$ length. The light attenuation losses in the simulation were below $0.5 \% / \mathrm{cm}$. For the full DarkMESA experiment, the simulation predicts a veto efficiency of more than $99.9 \%$ when employing this design. For the study of the remaining backgrounds at DarkMESA a preliminary concept exists based on (1) the analysis of beamoff data and (2) beam measurements with rotated detector geometries. This design for a veto system in the search for dark matter at accelerators follows the approach of the BDX Experiment at the Thomas Jefferson National Accelerator Facility (JLab) in the USA $[14,15]$.

This paper describes studies of prototype counters for the DarkMESA veto system in the $855-\mathrm{MeV}$ electron beam of the Mainz Microtron (MAMI). A scintillation counter, in which different configurations of fibers were embedded, is described in Section 2, the electron beam tests are presented in Section 3, the calibrations in Section 4, the light collection is discussed and modeled as a function of the transverse distance from the beam position to the fibers in Section 5, and the conclusions are given in Section 6 .

\section{Description of the scintillation counters}

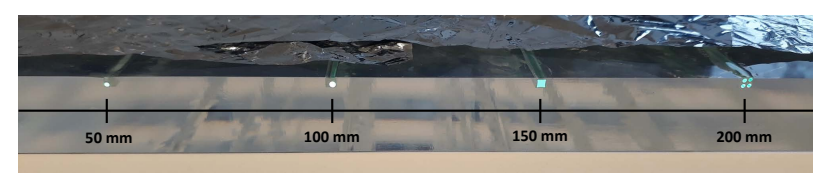

Figure 1: Photograph of one of the two read-out ends of the studied scintillation counter with fibers after polishing. Configurations from left to right: round fiber of 1-mm diameter, round fiber of $1.5-\mathrm{mm}$ diameter, square fiber of $2-\mathrm{mm}$ edge length, $2 \times 2$ matrix of four round fibers of 1 -mm diameter each.

Two identical scintillation counters of type EJ-200 from Eljen Technology [16] were used in this study. They had a size of $50 \times 25 \times 2 \mathrm{~cm}^{3}$ and highly polished surfaces to promote total internal reflection. 


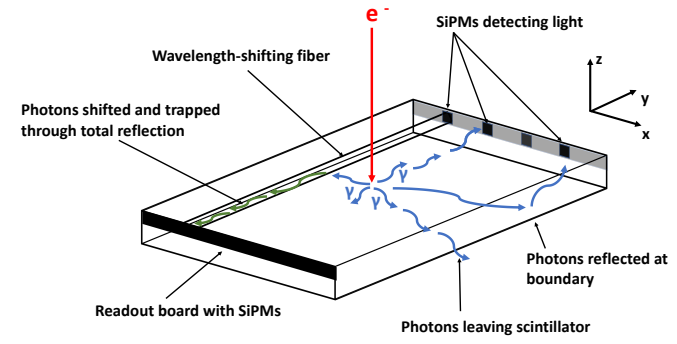

Figure 2: Schematic view of how a fiber is influencing the collection of scintillation light that is produced by a minimum ionizing electron beam penetrating the active volume of a counter. The scintillation counters in this study had a size of $50 \times 25 \times 2 \mathrm{~cm}^{3}$. The light was read out using four SiPMs mounted on a readout board on each of the two opposing ends.

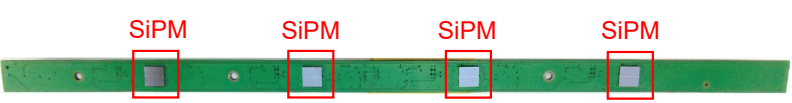

(a) Top layer

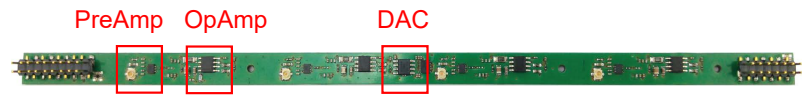

(b) Bottom layer

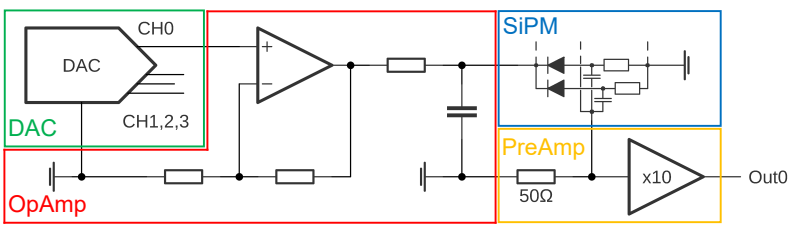

(c) Electronic circuit diagram

Figure 3: Photographs of the top and bottom layer of the readout board and the electronic circuit diagram. The board has a size of $1 \times 25 \mathrm{~cm}^{2}$. (a) Top layer. Four SiPMs with $6 \times 6 \mathrm{~mm}^{2}$ active area, and a capacitively coupled fast output. (b) Bottom layer. PreAmp (one per SiPM): Signal preamplifier based on the gain block AD8354 with a transimpedance gain of $Z=500 \Omega$ and high analog bandwidth; OpAmp (one per SiPM): Non-inverting high-voltage operational amplifier circuit with current-limiting resistor for generating the bias voltage from an adjustable reference voltage; DAC (one per board): Digital-to-analog converter for setting the individual values for the reference voltages and thus the bias voltages. (c) Electronic circuit diagram.
The opposite ends of the counters were each read out by four independent $6 \times 6 \mathrm{~mm}^{2} \mathrm{SiPMs}$ of type J-Series 60035 from SensL [17]. The active area of the SiPMs provide a sufficiently large coverage of the read-out ends of the counter without fibers. Parallel grooves of $2.5 \times 2.5 \mathrm{~mm}^{2}$ cross section were milled into the surface of one counter, so that fibers could be placed into these grooves, which were then filled with optical cement of type EJ-500 from Eljen Technology. The protruding ends of the fibers were cut off and the two readout sides of the counter were polished a second time. A photograph of one of the finished readout ends with the fibers can be seen in Fig. 1. The fibers were read out with the same type of SiPM. For this counter, a smaller size of the SiPM active area would have decreased noise as well as the detection of light not coming from the fiber, but would have also complicated the precise alignment to the groove and the comparison with the reference counter.

Four different fiber configurations were realized:

Ch0 Round WLS fiber of 1-mm diameter of type BCF-92 from Saint Gobain Crystals [18]

Ch1 Round WLS fiber of 1.5-mm diameter of type Y-11 (200) MJ from Kuraray [19]

Ch2 Square scintillating fiber of 2-mm edge length of type BCF-20 from Saint Gobain Crystals [18]

Ch3 $2 \times 2$ matrix of four round WLS fibers of 1-mm diameter each, bundled together, of type BCF92 from Saint Gobain Crystals [18]

The fibers from Kuraray are based on a polystyrene core with a refractive index of $n=1.59$, while Saint Gobain specifies for the core material $n=1.60$. All three different fibers have a thin inner cladding made from polymethylmethacrylate (PMMA) with $n=$ 1.49. The efficiency for trapping emitted light within the core of the round fibers ranges from $\epsilon=3.4 \%$ for light originating at the fiber axis to $\epsilon \sim 7 \%$ for light originating near the core-cladding interface. For square fibers, $\epsilon=4 \%$, independent of the point of origin. All fibers had a second outer cladding with $n=1.42$, increasing the core trapping efficiencies by about a factor of 1.6. The light emission in the scintillating fiber of channel Ch3 can also be excited by the 
blue light of the scintillation counter, so that it can act analogous to a WLS fiber. The peak emission wavelength of the different fibers is relatively similar: $476 \mathrm{~nm}$ for Y11 and $492 \mathrm{~nm}$ for BCF-92 and BCF-20. Based on the comparable light collection properties, the differences between the fibers can only marginally influence the light collection as a function of the transverse distance from the beam position to the fibers.

A schematic view of the readout concept for the scintillation light is presented in Fig. 2. On the readout board four individual SiPMs were powered each by an operational amplifier generating the bias voltage from an adjustable reference voltage. These voltages could be set by a digital-to-analog converter through SPI protocol by the slow control software. The SiPMs from SensL [17] feature a fast output signal with a significantly smaller capacitance than the anode output and an intrinsic FWHM pulse width of $3 \mathrm{~ns}$, allowing for precision timing and high count rates. All SiPMs were operated at a fixed bias voltage of $V_{\text {bias }}=27.3 \mathrm{~V}$, corresponding to an overvoltage of $V_{\mathrm{OV}} \sim 3 \mathrm{~V}$ and leading to a typical gain of $3 \times 10^{6}$. Note, that the temperature coefficient of the breakdown voltage is $d V_{\mathrm{BD}} / d T=21.5 \mathrm{mV} \mathrm{K}^{-1}$. The dark count rate of a single $6 \times 6 \mathrm{~mm}^{2} \mathrm{SiPM}$ is about $64 \mathrm{kHz} \mathrm{mm}^{-2}$ at $V_{\mathrm{OV}}=3 \mathrm{~V}$. The exact active area is $6.07 \times 6.07 \mathrm{~mm}^{2}$, the active area of each microcell is $35 \times 35 \mathrm{\mu m}^{2}$, and there are 22292 microcells in a sensor. The quoted fill factor is $75 \%$. The SiPMs feature a photon detection efficiency (PDE) of $40 \%$, an afterpulsing probability of $1 \%$, and a cross-talk probability of $10 \%$ at $V_{\mathrm{OV}}=3 \mathrm{~V}$. The outputs were connected to a signal preamplifier based on the gain block AD8354 with a transimpedance gain of $Z=$ $500 \Omega$ and high analog bandwidth, leading to an internal amplification factor of $G_{\text {int }}=10$ at $Z=50 \Omega$ input impedance. The output signals were split into a timing branch and a charge collection branch. The trigger signal for the data acquisition was realized with external electronic modules by discriminating the analog sum of the signals in the timing branch. The trigger threshold was set to $30 \mathrm{mV}$, corresponding to $15 \%$ of the most probable signal amplitude of a fully penetrating minimum-ionizing particle. The individual charge signals were externally amplified by another factor of $G_{\text {ext }}=10$. The signals of the SiPMs had a typical FWHM of $14 \mathrm{~ns}$ and a width of $30 \mathrm{~ns}$ at the base, and were integrated during a $70 \mathrm{~ns}$ long gate by a charge-sensitive ADC of type 2249A from LeCroy with a sensitivity of $0.25 \mathrm{pC}$ per ADC channel.

The readout board with the SiPMs and the frontend electronics are shown in Fig. 3. These boards were pushed onto the ends of the scintillation counters by a mechanical support. Optical grease was used to ensure optimal coupling of the SiPMs with these ends. The active area of the SiPMs of $6 \times 6 \mathrm{~mm}^{2}$ covered the grooves of $2.5 \times 2.5 \mathrm{~mm}^{2}$. The remaining area of the SiPMs was not optically shielded from the scintillator. To increase the collection of light, the scintillation counter was wrapped with aluminum-coated Mylar foil on all sides except the read-out sides.

\section{Electron beam tests of the scintillation counters}

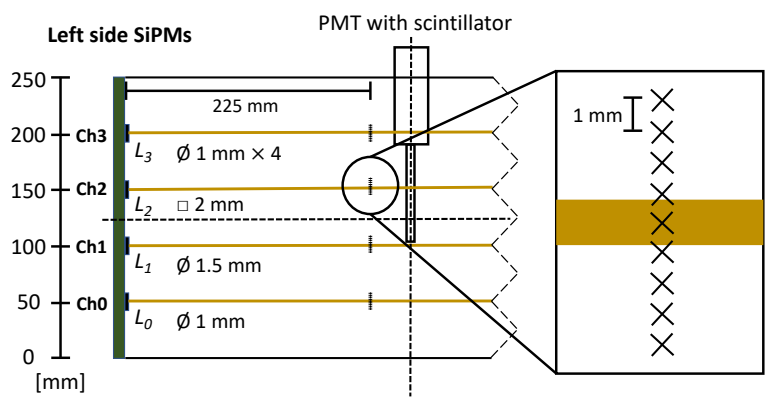

Figure 4: Positions of the electron beam (black crosses) on the 250-mm wide scintillation counter with fibers separated by $50 \mathrm{~mm}$. Near each fiber nine positions with a pitch interval of $1 \mathrm{~mm}$ were scanned at a distance of $225 \mathrm{~mm}$ from the readout side. The two dashed, perpendicular lines indicate the symmetry axes of the counter. The SiPMs of the four channels $\mathrm{Ch} 0$ to $\mathrm{Ch} 3$ at the left side are labeled $L_{0}$ to $L_{3}$. On the right, the relative scanning positions with respect to the fiber are shown in the enlarged view. The position of a separate, $5 \mathrm{~mm}$-wide scintillator in the center position of the counter is indicated.

Electron beams of $855 \mathrm{MeV}$ energy and subpicoampere currents from the Mainz Microtron 


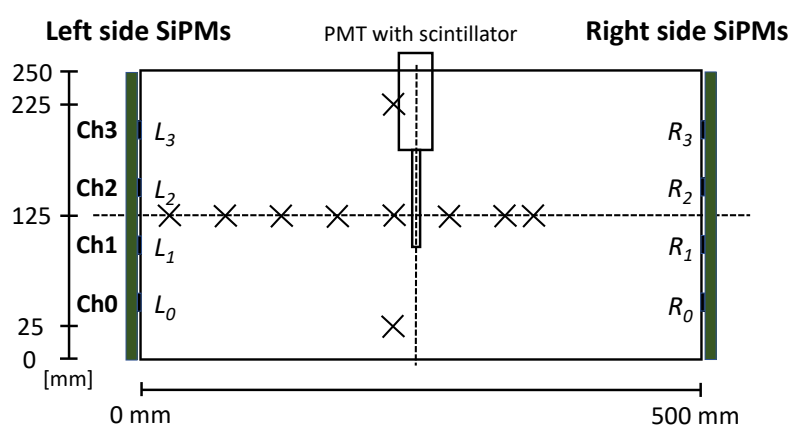

Figure 5: Positions of the electron beam (black crosses) on the $250-\mathrm{mm}$ wide scintillation counter without fibers for the reference measurements. Three positions with a pitch interval of $100 \mathrm{~mm}$ were scanned at a distance of $225 \mathrm{~mm}$ from the readout side. Seven additional measurements were taken along the central longitudinal axis. The two dashed, perpendicular lines indicate the symmetry axes of the counter. The SiPMs of the four channels $\mathrm{Ch} 0$ to $\mathrm{Ch} 3$ at the left and the right side are labeled $L_{0}$ to $L_{3}$, respectively $R_{0}$ to $R_{3}$. The position of a separate, $5 \mathrm{~mm}$-wide scintillator in the center position of the counter is indicated.

MAMI were precisely pointed to a set of positions on the top of one of the scintillation counters. The beam electrons deposited $\Delta E \approx 4 \mathrm{MeV}$ energy in the scintillator bulk, leading to $\mathcal{L} \sim 40000$ scintillation photons at the site of the ionizations. The typical analog sum of all eight SiPM output signals had amplitudes of $200 \mathrm{mV}$. The environmental temperature in the experiment was stabilized to minimize voltage drifts. Noise amplitudes were well below the trigger threshold and the trigger efficiency was estimated to be very close to $100 \%$. The measurements had a duration of $60 \mathrm{~s}$ at a data acquisition rate of $2 \mathrm{kHz}$ resulting in more than $10^{3}$ events in each charge spectrum. At these trigger rates, cosmogenic events and beam-unrelated background in the data set could be safely neglected.

The detector was placed in a dark box to shield it from external light sources and the whole setup was supported by a remotely steerable $x-y$ table. The beam left the vacuum beam pipe through an aluminum flange of $\Delta z \sim 0.2 \mathrm{~mm}$ thickness and traversed $z \sim 500 \mathrm{~mm}$ of air before hitting the detectors. Multiple scattering in the flange increased the divergence of the beam to $\theta_{\text {beam }}<1 \mathrm{mrad}$, result- ing in a beam spot of $\sigma_{\text {beam }} \ll 1 \mathrm{~mm}$ width at the position of the counters. The beam position along the longitudinal axis was determined by a $5 \mathrm{~mm}$-wide, separate scintillation detector coupled to a photomultiplier tube and located at the center position of the studied counters. The precision for the position with respect to the beam can be conservatively estimated with the detector width. The position on the transverse axis was determined by scanning the counter to its upper edge. This allowed to determine its position with respect to the beam with a precision of $\delta x \leq 1 \mathrm{~mm}$.

To study the light collection of the different configurations as a function of the transverse distance from the electron beam to a fiber, a scan parallel to the read-out side of the counter was performed as depicted in Fig. 4. The beam position relative to the fibers was determined from the scans with submillimeter precision. For reference, corresponding measurements were performed with the scintillation counter without embedded fibers. Three positions of the electron beam at the same distance from the read-out side were scanned as seen in Fig. 5.

\section{Calibration of the charge spectra}

\subsection{Evaluation of the number of photoelectrons}

Calibrations were performed in a separate study immediately after the beam tests under the same environmental conditions, in particular the same temperature. To convert the ADC values into a number of p.e., each SiPM was exposed to short LED light pulses at a wavelength of $450 \mathrm{~nm}$. The custommade LED and pulser setup featured a small pulse-topulse variation. The LED light was optically diffused $40 \mathrm{~cm}$ in front of the SiPMs and guaranteed a Poisson distributed number of photons per pulse sufficiently large to be in the Gaussian limit. Consequently, the resulting charge spectra showed symmetric, Gaussian distributed peaks with relative widths of the order of $10 \%$. If one assumes that the width of such a peak is caused by statistical fluctuations only, it will follow that $\sigma / \hat{n}=\sqrt{\lambda} / \lambda=1 / \sqrt{\lambda}$, where $\hat{n}$ is representing the position of the peak maximum, $\sigma$ the peak width, and $\lambda$ being the mean and variance of the Poisson distribution for the number of p.e. The electronic noise 
was determined independently from the LED by a pulser with a repetition frequency of $80 \mathrm{~Hz}$ and was significantly smaller than the peak widths. Including the subtraction of the measured pedestals in the charge spectra leads to the relation:

$$
\frac{\sqrt{\sigma_{\mathrm{LED}}^{2}-\sigma_{\mathrm{ped}}^{2}}}{\hat{n}_{\mathrm{LED}}-\hat{n}_{\mathrm{ped}}}=\frac{1}{\sqrt{\lambda}},
$$

where $\hat{n}_{\text {ped }}$ and $\sigma_{\text {ped }}$ are the position and width of a fit to the pedestal peak with a Gaussian distribution.

The conversion factors $c_{i}$ of calibrated ADC channels per p.e. were determined for each SiPM at a wide range of bias voltages from the calibrated peak positions $c_{i}=\left(\hat{n}_{\mathrm{LED}}-\hat{n}_{\text {ped }}\right) \cdot \kappa_{i} / \lambda$. The damping factors $\kappa_{i}$ needed to be included for each one of the eight SiPMs to account for signal losses through the cable pathways. They were determined by sending a well-defined amount of charge in pulses of a high precision frequency generator of type 81160A from Keysights Technologies through the signal pathways to the ADC.

The breakdown voltages $V_{\mathrm{BD}}$ of each SiPM were determined from this series of measurements by the projection of the measured gain to zero. The spread in breakdown voltages was up to $0.5 \mathrm{~V}$, in good agreement with the range specified in the data sheet.

\subsection{Evaluation of the light yield}

Figure 6 shows typical ADC spectra of a single SiPM, recorded when the scintillation light was produced by the electron beam penetrating the counter. The asymmetric peak shape could be well described by a Landau distribution to describe the physical distribution of the energy loss, convoluted with a Gaussian distribution with width parameter $\sigma$ to incorporate noise and other smearing effects. The most probable value $\hat{n}_{\mathrm{MPV}}$ of this function was used as a measure for the light yield, where the mean number of p.e. is given by $\lambda=\left(\hat{n}_{\mathrm{MPV}}-\hat{n}_{\text {ped }}\right) \cdot \kappa_{i} / c_{i}$. The ADC pedestals were determined in separate measurements.

The light yield $\lambda$ will be given in units of p.e. in this paper. A conversion to the number of incident photons $Y$ in units of ph. is wavelength-dependent and

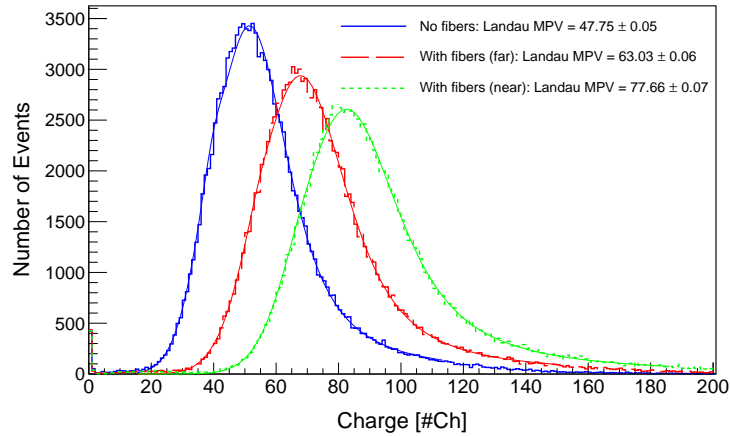

Figure 6: Typical ADC spectra $(\# \mathrm{Ch} \widehat{=} 0.25 \mathrm{pC})$ without calibration for a single SiPM recorded when the minimum ionizing electron beam penetrated the counter in a distance of $225 \mathrm{~mm}$ from the read-out side. For the left spectrum, a scintillation counter without embedded fibers was used. The most probable value of this distribution corresponds to a light yield of $\lambda \simeq 25$ p.e. after calibration. The center and right spectra were taken with a fiber of round geometry with diameter of $1.5 \mathrm{~mm}$ in a transverse distance of $4 \mathrm{~mm}$ (near) and $104 \mathrm{~mm}$ (far) to the beam position, respectively. The asymmetric peak shape could be well described by a Landau distribution convoluted with a Gaussian distribution.

may imply additional uncertainties. The number of discharged microcells in a SiPM for a light pulse of moderate intensity is proportional to its PDE. It depends further on the sum $q$ of the correlated noise of cross-talk and afterpulsing in the time window of the gate, leading to $Y=\frac{1-q}{P D E} \lambda$. The individual conversion factors for each SiPM were determined by a linear interpolation of the data sheet values for the PDE and the cross-talk plus afterpulsing probabilities to $V_{\mathrm{OV}} \sim 3 \mathrm{~V}$. They were typically on the order of $2.4 \mathrm{ph} . / \mathrm{p}$.e. for the blue light of the scintillation counters and $20 \%$ smaller for the light emitted by the fibers, with a larger reduction for the BCF fibers than for the Kuraray fiber.

\section{Analysis and modeling of the light yield}

\subsection{Reference light yield from a counter without fibers}

For a counter without fibers, the measured light yield was almost constant for beam positions along the transverse axis: the four inner SiPMs (Ch1 and 
Ch2, left and right) showed a variation of less than $1 \%$, while the four outer SiPMs (Ch0 and Ch3, left and right) showed a decrease or increase of not more than $3 \%$. These observations could be explained by light being produced in a thin counter that will get distributed almost homogeneously over the volume due to the many internal reflections. The average over the channels and transverse positions of the most probable light yields observed on the individual SiPMs was determined to be $\lambda_{\text {ref }}=(23.0 \pm 0.2)$ p.e. and then used as a reference value for the light yield for a counter without fibers.

The effective attenuation of the light produced along the central longitudinal axis was determined by the eight measurements indicated in Fig. 5. For beam positions at distances of more than $17 \mathrm{~cm}$ from the read-out side, no significant difference in light yield between the four channels of either side was found. At these positions the sum of the light yield from the corresponding two SiPMs on both sides was almost constant. The observed attenuation in longitudinal direction was less than $0.5 \% / \mathrm{cm}$. Single exponential fits to the light yield at more than $17 \mathrm{~cm}$ from the read-out sides resulted in an average effective light attenuation length of $\Lambda_{\text {att }}=220 \mathrm{~cm}$. The light sharing between the two sides, expressed as an asymmetry $\mathcal{A}=\left(L_{k}-R_{k}\right) /\left(L_{k}+R_{k}\right)$ for a channel $\mathrm{Ch} k$, allowed to determine the longitudinal position with a precision of $\delta z \sim 25 \mathrm{~mm}$.

\subsection{Light yield from a counter with fibers}

Figure 7 shows the light yield from a counter with fibers as a function of the transverse position of the beam, measured at a longitudinal distance of $225 \mathrm{~cm}$.

For all channels, the light yield across the transverse width of the counter showed a broad peak on top of a wide distribution, with the maximum position of the peak at the respective fiber position. As each fiber was placed in a groove of $2.5 \mathrm{~mm}$ depth, the reduced thickness of the scintillating material implied a local decrease in light yield at these positions. In case of the square fiber, the expected scintillation of the fiber was observed for direct electron beam exposure.

To model these observations, different descriptions of the wide distribution, the broad peak, and the local

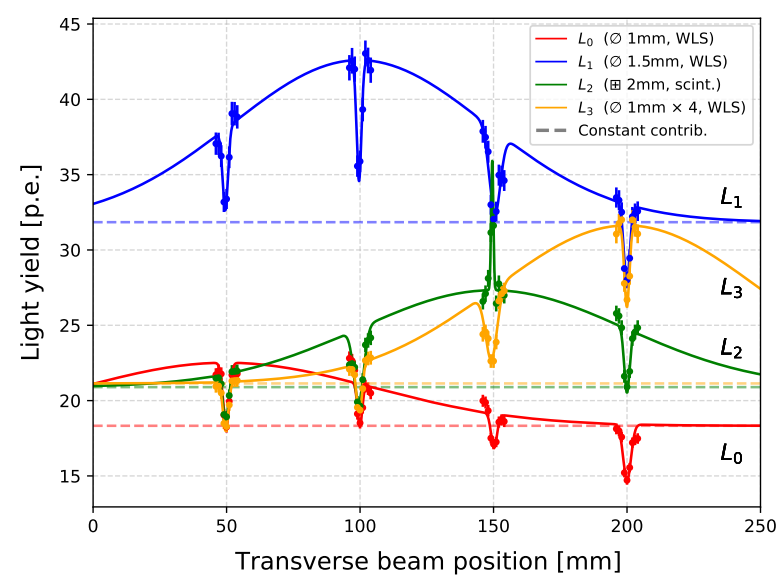

(a) Left end SiPMs

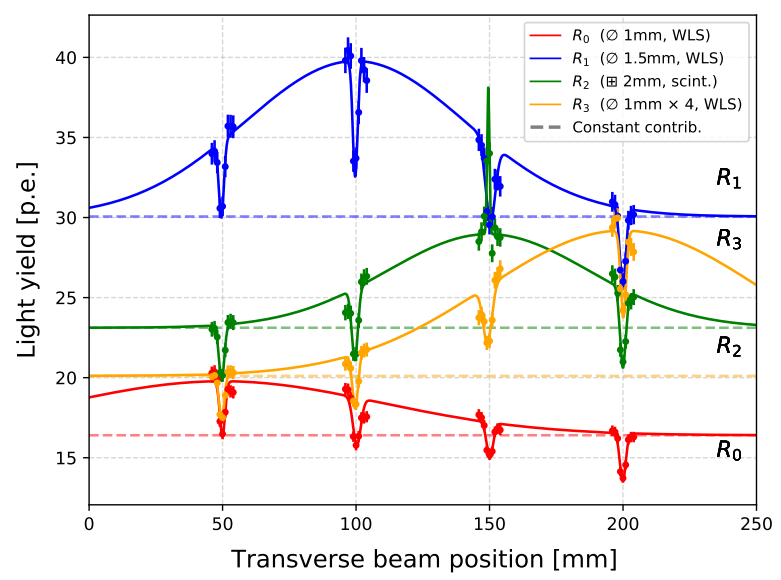

(b) Right end SiPMs

Figure 7: Light yield in number of photoelectrons for each SiPM connected to a fiber as a function of the transverse position of the beam. (a) Left end SiPMs. (b) Right end SiPMs. A broad peak on top of a wide distribution can be observed, with the maximum of the peak at the respective fiber position, where the beam was located. The effect of the grooves on the light yield is visible. The curves show a model description for the total light yield (full line), consisting of a constant (dashed line), local structures at the groove positions, and a peaking contribution. 
structures at the groove positions were tested.

It was found, that the wide distribution was best described by a constant. This is in agreement with the study of the counter without fibers, where no significant variation in light yield was observed between the four SiPMs on either side.

To describe the local structures at the groove positions, a sinc function, the second derivative of a Gaussian function, and a Gaussian function were tested. The nominal position of each fiber was determined by taking the mean value of all eight extremal positions from both ends, left and right, of the counter.

A well-motivated position dependence for the broad peak could be derived from the solid angle coverage of a fiber for direct light. This leads to the proportionality $\left.\Delta \Omega \propto 1 / \sqrt{(} x^{2}+z^{2}\right)$ with $x$ as transverse distance of the fiber from the light source and $z$ as the depth of the light source inside of the scintillator. After integration over $z$ from 0 to the thickness of the scintillator, this function shows a steep dependence close to the fiber. However, for larger distances, this dependence becomes inconsistent with the data. At these distances, a solid angle coverage approximated by $\Delta \Omega \propto 1 /\left(x^{2}+z^{2}\right)$, corresponding to an infinitely long fiber, provides a better description. A combination of both approaches leads to two complications: (1) With additional parameters the fit results are less robust and (2) the interpretation of the interplay of several effects or approximations in a sophisticated model becomes very difficult and less reliable. In contrast, a Gaussian or a Lorentz distribution can describe the broad peak reasonably well and provide at the same time (1) a very robust fit and (2) have only few parameters, that all have a clear interpretation. To conclude, some sophisticated models can provide a superior description of the data points in certain ranges compared to simpler functions, but fail in other ranges and add unnecessary complexity.

The best simultaneous fit to all data points from a channel $\mathrm{Ch} k$ was found with a linear combination of a constant yield $\lambda_{\text {far }}^{k}$, four Gaussian distributions at the four groove positions $\mu_{0-3}$, and one Gaussian distribution for the peaking contribution $\lambda_{\text {peak }}^{k}$ at the respective fiber position $\mu_{k}$ :

$$
\lambda^{k}(x)=\lambda_{\text {far }}^{k}+\lambda_{\text {peak }}^{k} \cdot \mathrm{e}^{-\frac{\left(x-\mu_{k}\right)^{2}}{2 \sigma_{k}^{2}}}+\sum_{i=0}^{3} \lambda_{\mathrm{i}}^{k} \cdot \mathrm{e}^{-\frac{\left(x-\mu_{i}\right)^{2}}{2 \sigma_{i}^{2}}}
$$

The observed light yields for the beam position near and far from the fiber, and a broad peak in the distribution, with $\lambda_{\text {near }}^{k}=\lambda_{\text {far }}^{k}+\lambda_{\text {peak }}^{k}$, motivated the naming of the parameters. This model resulted in a statistically acceptable $\chi^{2}$ for a reasonably low number of fitted parameters, where the number of degrees of freedom (n.d.f. $=21$ ) equals the number of scanned beam positions minus the number of fitted parameters. The interesting parameter values and the $\chi^{2} / n$.d.f. as a measure for the goodness of the fit are listed in Table 1.

\subsection{Systematic uncertainties and bias}

Systematic uncertainties of the light yields between channels could arise from the opto-mechanical coupling of the sensors with the readout sides of the counter. Such channel-to-channel uncertainties were estimated to be on the order of a few percent. Sources for shifts up or down of all light yields leading to correlated systematic uncertainties were not considered in this study. Factors contributing to a bias in the comparison of the light yield between channels could be due to different fiber characteristics such as different trapping efficiencies or attenuations in the fibers, their spectral matching with the SiPMs, or small temperature variations leading to different overvoltages of the SiPMs.

The systematic uncertainties of the light yields from the fitting and calibration procedures within one channel were estimated with 1 to $2 \%$. This range was evaluated from the variation of the residua of different model predictions and data points as well as the robustness of the fit results with respect to changes in the data and the calibrations. In the further analysis, the quoted uncertainties account for these point-topoint uncertainties within one channel as these are of relevance in the context of this study.

\subsection{Interpretation of the results}

The total light yield of the counter with fibers can be interpreted as composed of two contributions: 
1. One contribution to the collected light has a very weak dependence on its point of origin.

2. The second contribution to the collected light has a strong and peaking dependence on its point of origin.

The total light yield depends on many technical and geometrical aspects, such as groove size, coverage of SiPM active area, etc., while the peaking contribution can be attributed purely to the fiber.

The first contribution could be explained in analogy to the case of the counter without fibers: A fiber collects a certain fraction of the indirect light, so that one contribution to the light yield from a fiber should be varying only weakly with respect to the transverse direction. Besides, the active area of the SiPMs outside of the groove cross section is exposed to the scintillator bulk and adds to the light yield. This contribution $\lambda_{\text {bulk }}$ was calculated by scaling the measured reference light yield from the counter without fibers by the uncovered SiPM active area in the counter with fibers. The second contribution could be explained by light directly emitted into the solid angle covered by a fiber. This contribution should steeply increase as the position of the light production gets closer to the fiber.

Table 2 shows the gains in light yield for the separated contributions. All fiber configurations improved the light yield, when the light was being produced close to the respective fiber position. The gain with respect to the reference counter without fibers and related to the direct light collection was quantified by $G_{\text {peak }}=\lambda_{\text {peak }} / \lambda_{\text {ref }}$. It was found to be in the order of $45 \%$ for the 1.5 -mm fiber and distinctively lower for the other fiber configurations. The gain with respect to the reference counter without fibers for the indirect contribution was analogously quantified by $G_{\text {far }}=\left(\lambda_{\text {far }}-\lambda_{\text {bulk }}\right) / \lambda_{\text {ref. }}$. The 1.5$\mathrm{mm}$ fiber was improving the collection of indirect light in the same order of magnitude as the collection of direct light. In the other configurations, the gain for the indirect light collection was significantly lower. An increase in light yield by using fibers is invariably linked with a loss of homogeneity of light collection in the transverse distance from the point of origin to the fibers, when the light production is located in sufficient distance from the read-out side. For completeness, the near gain was defined as $G_{\text {near }}=G_{\text {far }}+G_{\text {peak }}$. Then, the difference between $G_{\text {near }}$ and $G_{\text {far }}$ for one fiber configuration quantifies the transverse inhomogeneity of the light yield imposed by the fibers. If the gains were calculated by interpolating the $\mathrm{SiPM}$ size to the groove size, using $G_{\text {far }}^{\text {matched }}=\left(\lambda_{\text {far }}-\lambda_{\text {bulk }}\right) /\left(\lambda_{\text {ref }}-\lambda_{\text {bulk }}\right)$ and the analogous definition for $G_{\text {peak }}^{\text {matched }}$, all gain values would be larger by a factor of $\lambda_{\text {ref }} /\left(\lambda_{\text {ref }}-\lambda_{\text {bulk }}\right)=5.75$. Relative to each other all values keep their proportion. Those gains provide a theoretical comparison of the light yield from a scintillation counter with fibers to a reference counter, where the SiPMs in both detectors have the size of the grooves, leading to the maximum possible gain that can be achieved with a wellchosen SiPM size. In practice, small-sized SiPMs are preferable for the detector with fibers and large-sized SiPMs are preferable for the detector without fibers.

\section{Conclusions}

This work has shown that, e.g., a WLS fiber with a round geometry and a diameter of $1.5 \mathrm{~mm}$ significantly increased the light yield from a box-shaped counter of $2 \mathrm{~cm}$ thickness in which it was embedded. For configurations with fibers of smaller diameter or made from a bundle of such fibers, the gain in light yield was distinctively smaller. It was observed that the gain increased strongly for a point of origin of the light within distances of $\pm 10 \mathrm{~cm}$ to the fiber. The data were modeled by attributing the sum of two contributions to the total light yield: the collection of indirect light and of direct light reaching a fiber. Based on the observation, one should place this type of fibers in veto counters such as the one for DarkMESA with a maximum distance of $10 \mathrm{~cm}$ to profit from the collection of direct light.

The dependence of the light yield on the point of origin of the light can lead to complications in the interpretation of the SiPM output signals in WLS fiber configurations. For example, in the BDX Experiment at JLab, a detailed description of the counter geometry and the photoelectron response needed to be implemented in a simulation framework to account for these complications [15]. On the other hand, the 
Table 1: Separated light yield contributions for a beam position far from the respective fiber $\lambda_{\text {far }}$, the broad peak $\lambda_{\text {peak }}$, and the sum of both with $\lambda_{\text {near }}=\lambda_{\text {far }}+\lambda_{\text {peak }}$, averaged for each pair of SiPMs from the left and the right side of a fiber. The last line gives the reference value $\lambda_{\text {ref }}$ from the counter without fibers. The quoted uncertainties account for point-to-point uncertainties within one channel.

\begin{tabular}{lrrrcc}
\hline Channel & $\lambda_{\text {far }}$ (p.e.) & $\lambda_{\text {peak }}$ (p.e.) & $\lambda_{\text {near }}$ (p.e.) & \multicolumn{2}{c}{$\chi^{2} /$ n.d.f. } \\
& & & & Left & Right \\
\hline Ch0 $(\varnothing 1 \mathrm{~mm})$ & $17.0 \pm 0.4$ & $3.5 \pm 0.5$ & $20.5 \pm 0.7$ & 3.1 & 2.2 \\
Ch1 $(\varnothing 1.5 \mathrm{~mm})$ & $30.6 \pm 0.5$ & $10.1 \pm 0.6$ & $40.7 \pm 0.8$ & 1.2 & 1.4 \\
Ch2 $(\boxplus 2 \mathrm{~mm})$ & $22.8 \pm 0.4$ & $6.0 \pm 0.4$ & $28.8 \pm 0.6$ & 1.8 & 1.5 \\
Ch3 $(\varnothing 1 \mathrm{~mm} \times 4)$ & $20.7 \pm 0.2$ & $9.9 \pm 0.4$ & $30.6 \pm 0.5$ & 1.2 & 1.7 \\
\hline Reference $($ no fibers $)$ & $23.0 \pm 0.2$ & & & & \\
\hline
\end{tabular}

Table 2: Gains in light yield $G_{\text {far }}=\left(\lambda_{\text {far }}-\lambda_{\text {bulk }}\right) / \lambda_{\text {ref }}, G_{\text {peak }}=\lambda_{\text {peak }} / \lambda_{\text {ref }}$, and $G_{\text {near }}=G_{\text {far }}+G_{\text {peak }}$ averaged for each pair of SiPMs from the left and the right side of a fiber, with respect to the reference value from the counter without fibers. The correction for the partial coverage of the bulk scintillator by the SiPM active area has been taken into account for $G_{\text {far }}$, which could add a systematic bias. The difference between $G_{\text {near }}$ and $G_{\text {far }}$ for one fiber configuration quantifies the transverse inhomogeneity of the light yield imposed by the fibers. All gains would be larger by a factor of 5.75 , if the SiPMs were covering only the groove, see text. The quoted uncertainties account for point-to-point uncertainties within one channel.

\begin{tabular}{lrrr}
\hline Channel & $G_{\text {far }}(\%)$ & $G_{\text {peak }}(\%)$ & $G_{\text {near }}(\%)$ \\
\hline Ch0 $(\varnothing 1 \mathrm{~mm})$ & $-8.9 \pm 1.7$ & $15.0 \pm 1.9$ & $6.2 \pm 2.6$ \\
Ch1 $(\varnothing 1.5 \mathrm{~mm})$ & $50.3 \pm 2.4$ & $43.9 \pm 2.3$ & $94.2 \pm 3.4$ \\
Ch2 $(\boxplus 2 \mathrm{~mm})$ & $16.1 \pm 1.7$ & $26.0 \pm 1.8$ & $42.1 \pm 2.5$ \\
Ch3 $(\varnothing 1 \mathrm{~mm} \times 4)$ & $7.1 \pm 1.2$ & $42.8 \pm 1.4$ & $49.9 \pm 1.9$ \\
\hline
\end{tabular}


transverse position sensitivity could have a positive effect, for instance to determine the point of origin within the counter with an increased resolution when considering signal intensities of multiple SiPMs. On the contrary, the longitudinal position resolution provided by the light sharing in a counter without fibers could also be an advantage.

The veto counters for the small DarkMESA prototype, that has a size of $50 \times 50 \times 80 \mathrm{~cm}^{3}$, were constructed without embedded fibers, retaining the relative ease of construction of the veto system. The design of the readout board was optimized for this application and incorporates nine instead of four SiPMs, thereby increasing the total light yield and improving the uniformity at the read-out ends. Future studies of its detection efficiency will focus, for instance, on the longitudinal homogeneity and possible edge effects.

\section{CRediT authorship contribution statement}

M. Lauß: Conceptualization, Formal analysis, Investigation, Methodology, Review \& Editing, Software, Visualization, Writing - Original Draft. P. Achenbach: Conceptualization, Formal analysis, Funding acquisition, Investigation, Methodology, Project administration, Review \& Editing, Supervision, Writing - Original Draft. S. Aulenbacher: Review \& Editing. M. Ball: Review \& Editing. I. Beltschikow: Investigation, Review \& Editing, Software. M. Biroth: Conceptualization, Formal analysis, Investigation, Methodology, Review \& Editing, Visualization. P. Brand: Review \& Editing. S. Caiazza: Review \& Editing. M. Christmann: Conceptualization, Investigation, Methodology, Review \& Editing. O. Corell: Resources, Review \& Editing. A. Denig: Funding acquisition, Project administration, Review \& Editing. L. Doria: Funding acquisition, Project administration, Review \& Editing. P. Drexler: Investigation, Review \& Editing, Software. J. Geimer: Review \& Editing. P. Gülker: Investigation, Review \& Editing. T. Kolar: Review \& Editing. W. Lauth: Conceptualization, Investigation, Resources, Review \& Editing. M. Littich: Review \& Editing. M. Lupberger: Review \& Editing. S. Lunkenheimer:
Review \& Editing. D. Markus: Review \& Editing. M. Mauch: Review \& Editing. H. Merkel: Funding acquisition, Project administration, Resources, Review \& Editing. M. Mihovilovič: Review \& Editing. J. Müller: Review \& Editing. B. S. Schlimme: Funding acquisition, Project administration, Review \& Editing. C. Sfienti: Funding acquisition, Review \& Editing. S. Širca: Review \& Editing. S. Stengel: Review \& Editing. C. Szyszka: Review \& Editing. S. Vestrick: Review \& Editing.

\section{Acknowledgments}

The authors would like to thank the MAMI operators, technical staff, and the accelerator group for their excellent work. We also thank P. L. Cole for language support.

This work was supported by the PRISMA ${ }^{+}$Cluster of Excellence "Precision Physics, Fundamental Interactions and Structure of Matter", and by the Helmholtz-Gemeinschaft Deutscher Forschungszentren (HGF) with a HGF-Exzellenznetzwerk.

\section{References}

[1] F. Hug, K. Aulenbacher, R. Heine, B. Ledroit, D. Simon, MESA — an ERL project for particle physics experiments, in: Proc. Linear Accelerator Conference (LINAC2016), East Lansing, MI, USA, 25 - 30 September 2016, 2017, pp. 313-315, https://doi.org/10.18429/JACoWLINAC2016-MOP106012.

[2] L. Doria, P. Achenbach, M. Christmann, A. Denig, P. Gülker, H. Merkel, Search for light dark matter with the MESA accelerator, in: Proc. 13th International Conference on the Intersection of Particle and Nuclear Physics (CIPANP18), Palm Springs, CA, USA, 29 May - 3 June 2018, to appear in eConf, 2018, http://arxiv.org/abs/1809.07168.

[3] L. Doria, P. Achenbach, M. Christmann, A. Denig, H. Merkel, Dark matter at the intensity frontier: The new MESA electron accelerator facility, in: Proc. An Alpine LHC 
Physics Summit 2019 (ALPS 2019), Obergurgl, Austria, 22 - 27 April 2019, vol. 360 of PoS Proc. Sci. (ALPS2019), 2021, p. 22, https://doi.org/10.22323/1.360.0022.

[4] J. D. Bjorken, R. Essig, P. Schuster, N. Toro, New fixed-target experiments to search for dark gauge forces, Phys. Rev. D 80 (2009) 075018, https://doi.org/10.1103/PhysRevD.80.075018.

[5] M. Christmann, P. Achenbach, S. Baunack, P. Burger, A. Denig, L. Doria, F. Maas, H. Merkel, Instrumentation and optimization studies for a beam dump experiment (BDX) at MESA - DarkMESA, Nucl. Instrum. Methods Phys. Res. A (2019) 162398, https://doi.org/10.1016/j.nima.2019.162398.

[6] V. Balagura, M. Danilov, B. Dolgoshein, S. Klemin, R. Mizuk, P. Pakhlov, E. Popova, V. Rusinov, E. Tarkovsky, I. Tikhomirov, Study of scintillator strip with wavelength shifting fiber and silicon photomultiplier, Nucl. Instrum. Methods Phys. Res. A 564 (2006) 590-596, https://doi.org/10.1016/j.nima.2006.04.030.

[7] V. Andreev, et al., A high granularity scintillator calorimeter readout with silicon photomultipliers, Nucl. Instrum. Methods Phys. Res. A 540 (2005) 368-380, https://doi.org/10.1016/j.nima.2004.12.002.

[8] C. Adloff, et al. (The CALICE Collaboration), Construction and commissioning of the CALICE analog hadron calorimeter prototype, J. Instrum. 5 (2010) P05004, https://doi.org/10.1088/17480221/5/05/P05004, http://arxiv.org/abs/1003.2662.

[9] M. Yokoyama, A. Minamino, S. Gomi, K. Ieki, N. Nagai, T. Nakaya, K. Nitta, D. Orme, M. Otani, T. Murakami, T. Nakadaira, M. Tanaka, Performance of multi-pixel photon counters for the T2K near detectors, Nucl. Instrum. Methods Phys. Res. A 622 (2010) 567-573, https://doi.org/10.1016/j.nima.2010.07.070, http://arxiv.org/abs/1007.2712.

[10] K. Abe, et al. (The T2K Collaboration), The T2K experiment, Nucl. Instrum. Methods Phys. Res. A 659 (2011) 106-135, https://doi.org/10.1016/j.nima.2011.06.067, http://arxiv.org/abs/1106.1238.

[11] F. Simon, Silicon photomultipliers in particle and nuclear physics, Nucl. Instrum. Methods Phys. Res. A 926 (2019) 85-100, https://doi.org/10.1016/j.nima.2018.11.042, http://arxiv.org/abs/1811.03877.

[12] D. Denisov, V. Evdokimov, S. Lukić, P. Ujić, Test beam studies of the light yield, time and coordinate resolutions of scintillator strips with WLS fibers and SiPM readout, Nucl. Instrum. Methods Phys. Res. A 848 (2017) 54-59, https://doi.org/10.1016/j.nima.2016.12.043.

[13] A. Artikov, V. Baranov, G. C. Blazey, N. Chen, D. Chokheli, Y. Davydov, E. C. Dukes, A. Dychkant, R. Ehrlich, K. Francis, M. Frank, V. Glagolev, C. Group, S. Hansen, S. Magill, Y. Oksuzian, A. Pla-Dalmau, P. Rubinov, A. Simonenko, E. Song, S. Stetzler, Y. Wu, S. Uzunyan, V. Zutshi, Photoelectron yields of scintillation counters with embedded wavelength-shifting fibers read out with silicon photomultipliers, Nucl. Instrum. Methods Phys. Res. A 890 (2018) 84-95, https://doi.org/10.1016/j.nima.2018.02.023.

[14] M. Battaglieri, et al. (The BDX Collaboration), Dark matter search in a BeamDump eXperiment (BDX) at Jefferson Lab, Proposal to the Program Advisory Committee 45, Thomas Jefferson Laboratory, 2018, https://doi.org/10.2172/1431583, http://arxiv.org/abs/1712.01518.

[15] M. Battaglieri, P. Bisio, M. Bondí, A. Celentano, P. L. Cole, M. De Napoli, R. De Vita, L. Marsicano, G. Ottonello, F. Parodi, N. Randazzo, E. S. Smith, D. SnowdenIfft, M. Spreafico, T. Whitlatch, M. H. Wood, 
The BDX-MINI detector for Light Dark Matter search at JLab, Eur. Phys. J. C 81 (2021)

164, https://doi.org/10.1140/epjc/s10052-02108957-5, http://arxiv.org/abs/2011.10532.

[16] Eljen Technology, Scintillator Properties, 2020, available at https://eljentechnology. com/products/plastic-scintillators/ ej-200-ej-204-ej-208-ej-212.

[17] SensL, J-Series Datasheet, 2020, available at http://sensl.com/downloads/ds/ DS-MicroJseries.pdf.

[18] Saint Gobain Crystals, Scintillation Products, 2020, available at http: //fixels.physics.ucsb.edu/Lgbk/pub/E41. dir/SGC_Scintillating_Optical_Fibers_ Brochure_605.pdf.

[19] Kuraray, Plastic Scintillating Fibres, 2020, available at http://kuraraypsf.jp/pdf/all.pdf. 